\title{
A Comparative Investigation of Several Frequency Modulation Profiles for Programmed Switching Controllers Targeted Conducted-Noise Reduction in DC-DC Converters
}

\author{
Gamal M. DOUSOKY ${ }^{\dagger a)}$, Student Member, Masahito SHOYAMA ${ }^{\dagger}$, and Tamotsu NINOMIYA ${ }^{\dagger \dagger}$, Members
}

\begin{abstract}
SUMMARY This paper investigates the effect of several frequency modulation profiles on conducted-noise reduction in dc-dc converters with programmed switching controller. The converter is operated in variable frequency modulation regime. Twelve switching frequency modulation profiles have been studied. Some of the modulation data are prepared using MATLAB software, and others are generated online. Moreover, all the frequency profiles have been designed and implemented using FPGA and experimentally investigated. The experimental results show that the conducted-noise spreading depends on both the modulation sequence profile and the statistical characteristics of the sequence. A substantial part of the manufacturing cost of power converters for telecommunication applications involves designing filters to comply with the EMI limits. Considering this investigation significantly reduces the filter size.

key words: DC-DC power conversion, electromagnetic compatibility, noise reduction, frequency modulation, programmed switching
\end{abstract}

\section{Introduction}

Switching power converters generate considerable electromagnetic interference (EMI) noise [1]. EMI noise reduction is generally accomplished by three means: suppression of noise source, isolation of noise coupling path, and filter/shielding [2]. Common tools such as passive filters and shields will put increased pricing pressures on dc-dc converters.

The noise peaks of EMI spectrum are mainly concentrated on multiples of switching frequency. However, in the case of switching frequency modulation techniques, the energy of harmonics is spread across a well defined frequency band; the peak of harmonics drastically decreases [2]-[15]. This paper investigates the effect of a dozen of frequency modulation profiles on conducted-noise reduction in dc-dc converters using a programmed switching controller. Considering this investigation can permit a significant saving in the required EMI filter cost.

The field-programmable gate array (FPGA) is a flexible and attractive hardware design option, becoming lower cost, and applicable for power supply applications [6][9]. The implementation of investigated spread-spectrum regimes has been accomplished by FPGA technology.

Manuscript received January 18, 2010.

Manuscript revised April 22, 2010.

$\dagger$ The authors are with Kyushu University, Fukuoka-shi, 8190395 Japan.

${ }^{\dagger}$ The author is with Nagasaki University, Nagasaki-shi, 8528521 Japan.

a)E-mail: gamal@ees.kyushu-u.ac.jp

DOI: 10.1587/transcom.E93.B.2265
This paper is organized as follows. Section 2 describes the programmed frequency modulation. Section 3 presents details of the experimental test circuit. Experimental results and discussions are presented in Sect. 4. Finally, conclusions are addressed Sect. 5 .

\section{Programmed Frequency Modulation}

The implementation of the studied profiles has been accomplished by using an FPGA-based controller and experimentally investigated as shown in Fig. 1.

\subsection{Investigated Frequency Modulation Profiles}

The investigated profiles can be classified into two groups as follows:

Group I:

1. Sinusoidal modulation.

2. Triangular modulation.

3. Sawtooth modulation.

4. Exponential modulation.

5. Square modulation.

6. Zigzag modulation.

Group II:

7. Chaotic modulation.

8. Markov chain based random modulation.

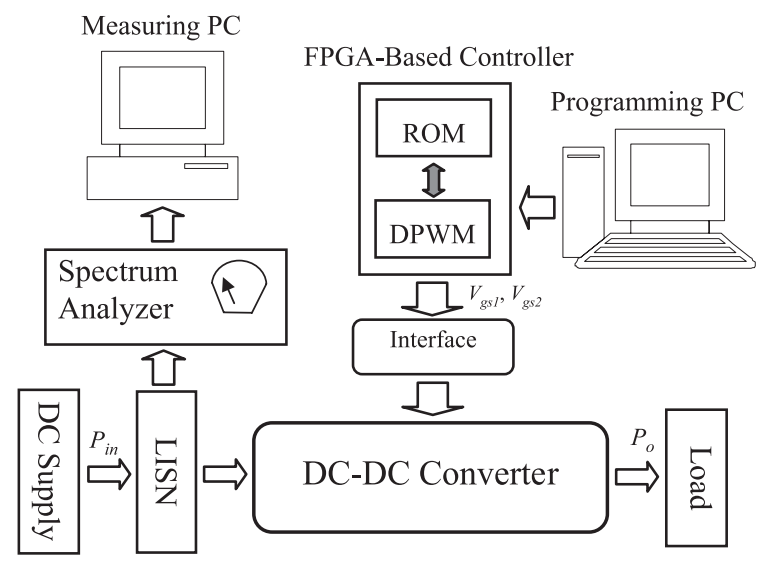

Fig. 1 Block diagram of the controlled DC-DC converter with the conducted-noise measurement system. 
9. Uniformly distributed random modulation.

10. Normally distributed random modulation.

11. M-sequence based random modulation.

12. Pseudorandom stream modulation.

\subsection{Data Preparation and Implementation}

All the investigated profiles fulfill the following considerations:

- Center switching frequency, $f_{\text {csw }}=300 \mathrm{kHz}$.

- Frequency deviation, $\Delta F= \pm 25 \%$, and so: Lower switching frequency limit, $f_{L}=0.75 \times 300=$ $225 \mathrm{kHz}$.

Upper switching frequency limit, $f_{H}=1.25 \times 300=$ $375 \mathrm{kHz}$.

- Number of the switching frequency points in the modulated sequence array, $N_{p}=256$ point.

All the twelve switching frequency modulation profiles, stated above, have been investigated. Some of the modulation data are prepared using MATLAB software, and the others are generated online. These profiles can be described in detail as follows:

\subsubsection{Sinusoidal Modulation}

The mathematical expression of the modulated frequency, following a sinusoidal modulation profile, is:

$$
F_{k}=\frac{\left(f_{H}-f_{L}\right)}{2} \sin \left(\frac{2 \pi k}{N_{p}}\right)+f_{c s w}
$$

where $F_{k}$ is the switching frequency of the kth cycle, and $k$ $=1,2, \ldots, N_{p}$.

Figure 2(a) shows the modulated switching frequency sequence with a sinusoidal profile. In addition, Fig. 2(b) presents the calculated discrete probability distribution of these frequencies and their probability mass level. The center frequency is swept from the lower frequency to the upper frequency. The presented probability mass level at the center frequency of the bandwidth is the sum of the discrete probability values at the frequencies that fall within the bandwidth. In order to obtain a meaningful discussion that concisely implies the relation between the measured noise spectra and the investigated profiles, the resolution bandwidth (RBW) is taken as the same RBW of the spectrum analyzer $(\mathrm{RBW}=9 \mathrm{kHz})$.

\subsubsection{Triangular Modulation}

The mathematical expression of the modulated frequency, following a triangular modulation profile, is:

$$
F_{k}=\left\{\begin{array}{l}
\left(f_{H}-f_{c s w}\right)\left(\frac{4 k}{N_{p}}\right)+f_{c s w}, \text { for } k \leq \frac{N_{p}}{4} \\
\left(f_{L}-f_{H}\right)\left(\frac{2\left(k-\frac{N_{p}}{4}\right)}{N_{p}}\right)+f_{H}, \text { for } \frac{N_{p}}{4}<k \leq \frac{3 N_{p}}{4} \\
\left(f_{c s w}-f_{L}\right)\left(\frac{4\left(k-\frac{3 N_{p}}{4}\right)}{N_{p}}\right)+f_{L}, \text { for } k>\frac{3 N_{p}}{4}
\end{array}\right.
$$

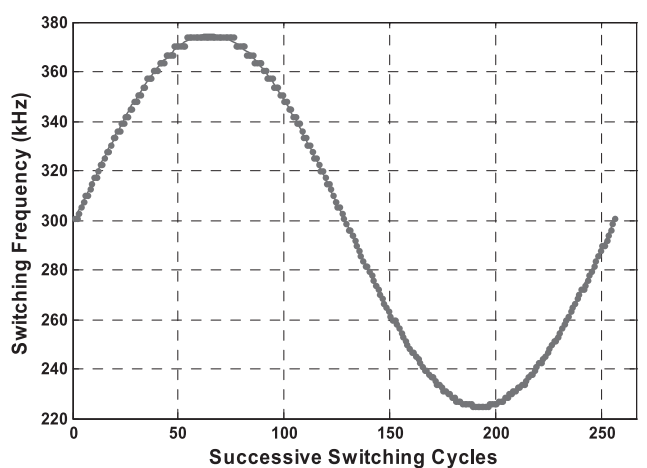

(a)

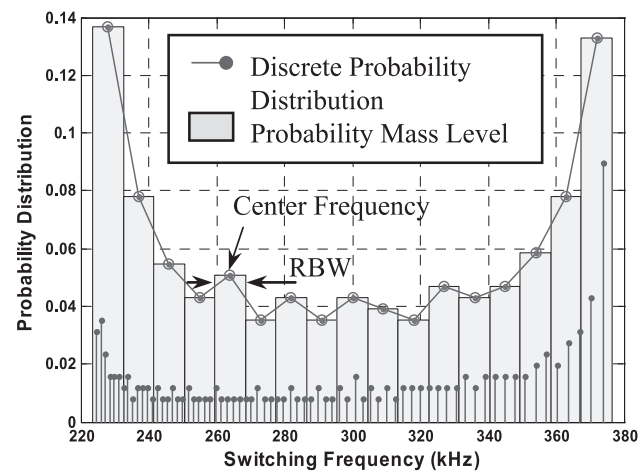

(b)

Fig. 2 Sinusoidal modulation: (a) modulated switching frequency sequence and (b) discrete probability distribution and probability mass level.

Figure 3 shows the modulated switching frequency sequence with a triangular profile and its probability characteristics.

\subsubsection{Sawtooth Modulation}

The mathematical expression of the modulated frequency, following a sawtooth modulation profile, is:

$$
F_{k}=\left\{\begin{array}{l}
\left(f_{H}-f_{c s w}\right)\left(\frac{2 k}{N_{p}}\right)+f_{c s w}, \text { for } k \leq \frac{N_{p}}{2} \\
\left(f_{c s w}-f_{L}\right)\left(\frac{2\left(k-\frac{N_{p}}{2}\right)}{N_{p}}\right)+f_{L}, \text { for } k>\frac{N_{p}}{2}
\end{array}\right.
$$

\subsubsection{Exponential Modulation}

The introduced exponential modulation profile in [10]-[12] is applied here for comparing the EMI performances. The mathematical expression of the modulated frequency, following an exponential modulation profile, can be expressed in a discrete form as: 


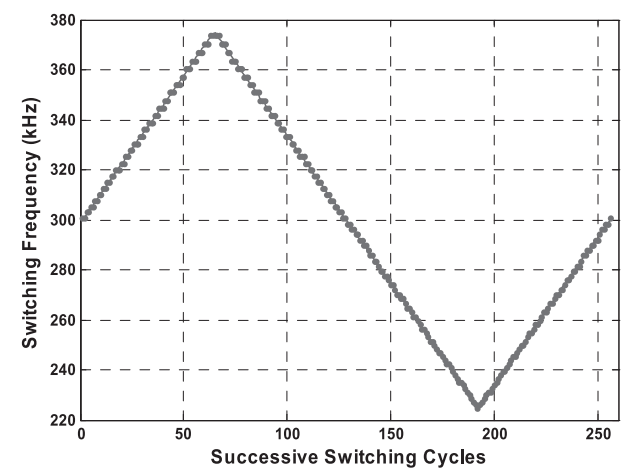

(a)

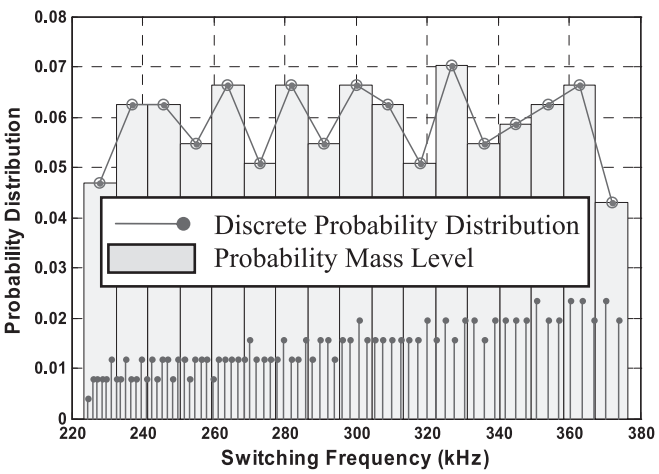

(b)

Fig. 3 Triangular modulation: (a) modulated switching frequency sequence and (b) discrete probability distribution and probability mass level.

$$
F_{k}=\left\{\begin{array}{l}
A_{p}\left(e^{\left(\frac{p^{k}}{N_{p}}\right)}-1\right), \text { for } k \leq \frac{N_{p}}{4} \\
A_{p}\left(e^{\left(\frac{p}{2 f_{m}}\right)} e^{\left(\frac{p^{k}}{N_{p}}\right)}-1\right), \text { for } \frac{N_{p}}{4}<k \leq \frac{N_{p}}{2} \\
A_{p}\left(1-e^{\left(\frac{-p}{2 f_{m}}\right)} e^{\left(\frac{p^{k}}{N_{p}}\right)}\right), \text { for } \frac{N_{p}}{2}<k \leq \frac{3 N_{p}}{4} \\
A_{p}\left(1-e^{\left(\frac{p}{2 f_{m}}\right)} e^{\left(\frac{p^{k}}{N_{p}}\right)}\right), \text { for } k>\frac{3 N_{p}}{4}
\end{array}\right.
$$

where $A_{p}=\frac{f_{H}-f_{c s w}}{\left(e^{\left(\frac{p}{4 f m}\right)}-1\right)}, p$ is a parameter controlling the concavity or convexty of the exponential profile. The results in this paper were obtained at a value of $p=12 f_{m}$.

Figure 4 shows the modulated switching frequency sequence with an exponential profile and its probability characteristics.

\subsubsection{Square Modulation}

The modulated switching frequency sequence with a square profile has been calculated using (4), at $p=-144 f_{m}$. Figure 5 shows the modulated switching frequency sequence with a square profile and its probability characteristics.

\subsubsection{Zigzag Modulation}

The mathematical expression of the modulated frequency, following a zigzag modulation profile, can be presented as:

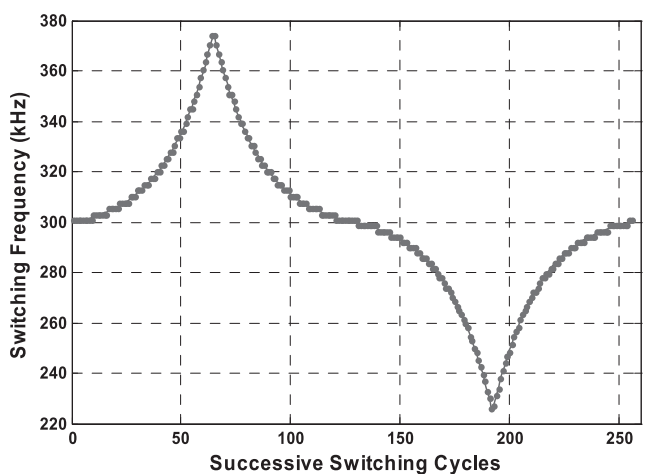

(a)

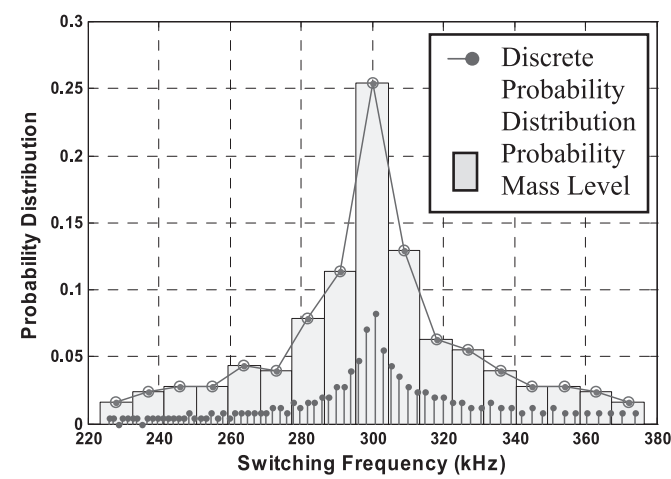

(b)

Fig. 4 Exponential modulation: (a) modulated switching frequency sequence and (b) discrete probability distribution and probability mass level.

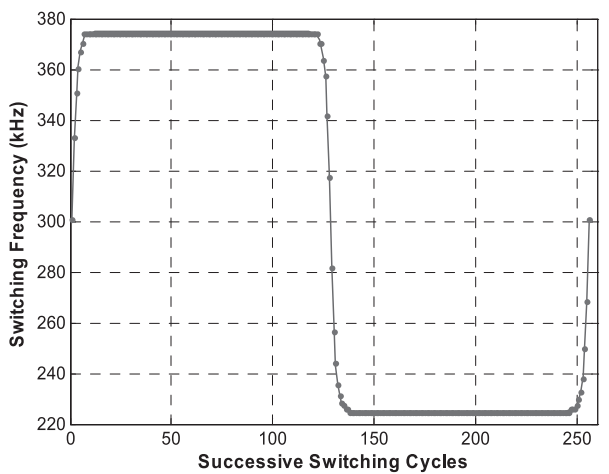

(a)

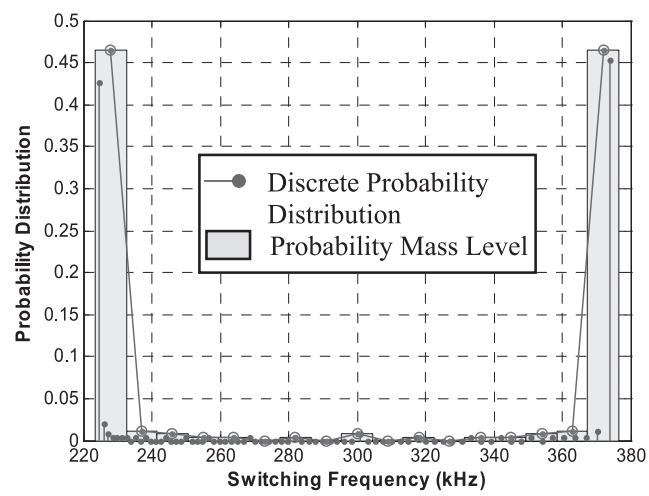

(b)

Fig. 5 Square modulation: (a) modulated switching frequency sequence and (b) discrete probability distribution and probability mass level. 


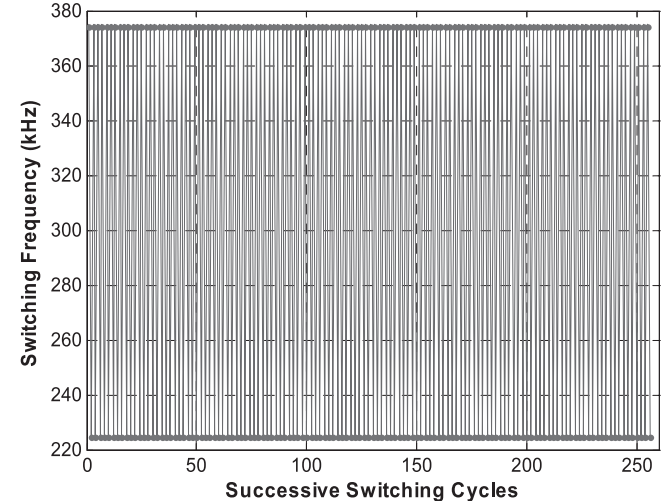

(a)

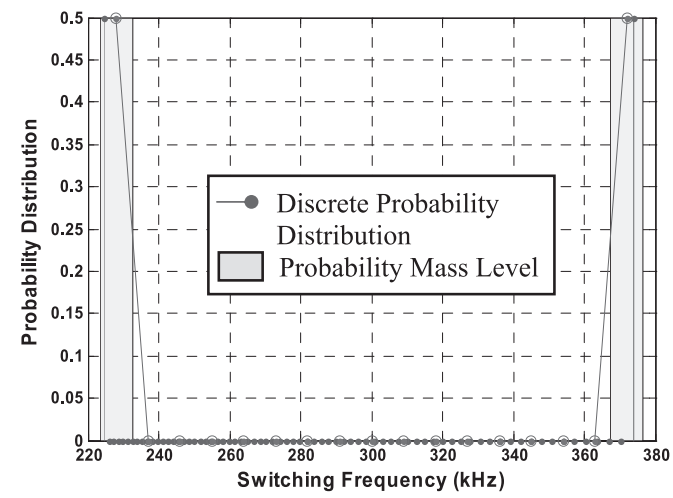

(b)

Fig. 6 Zigzag modulation: (a) modulated switching frequency sequence and (b) discrete probability distribution and probability mass level.

$$
F_{k}=\left\{\begin{array}{l}
f_{H}, \text { for odd } k \\
f_{L}, \text { for even } k
\end{array}\right.
$$

Figure 6 shows the modulated switching frequency sequence with a zigzag profile and its probability characteristics. It is noticed that the probability mass level of such profile (seen in Fig. 6(b)) is similar to that in the previous case (square modulation, seen in Fig. 5(b)).

\subsubsection{Chaotic Modulation}

The mathematical expression of the modulated frequency, following a chaotic modulation profile, can be presented as:

$$
F_{k}=\left(f_{H}-f_{L}\right) \times\left|\sin \left(\frac{N_{p}}{c k}\right)\right|+f_{L}
$$

where $c$ is a parameter controlling the distribution of the chaotic profile. The results in this paper were obtained at a value of $c=0.001$.

Figure 7 shows the modulated switching frequency sequence with a chaotic profile and its probability characteristics.

\subsubsection{Markov Chain Based Random Modulation}

In this case, the modulated frequency is chosen according to

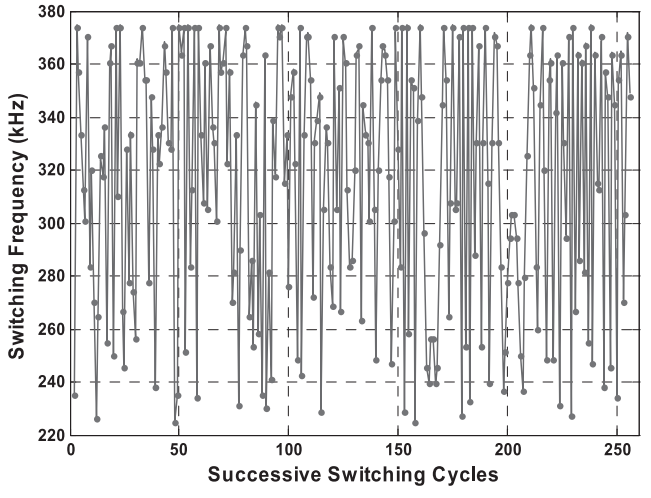

(a)

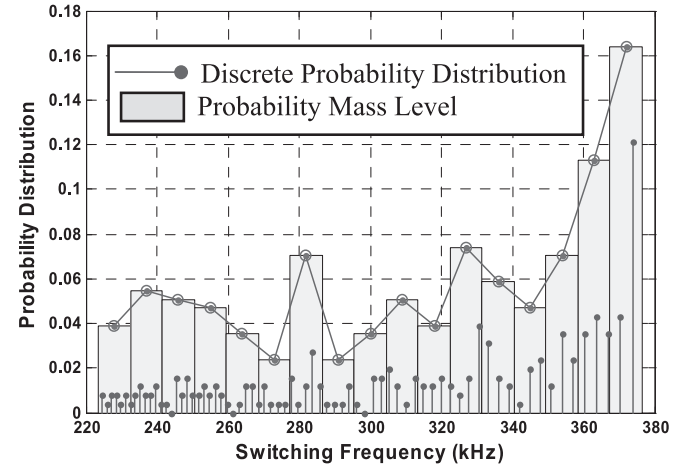

(b)

Fig. 7 Chaotic modulation: (a) modulated switching frequency sequence and (b) discrete probability distribution and probability mass level.

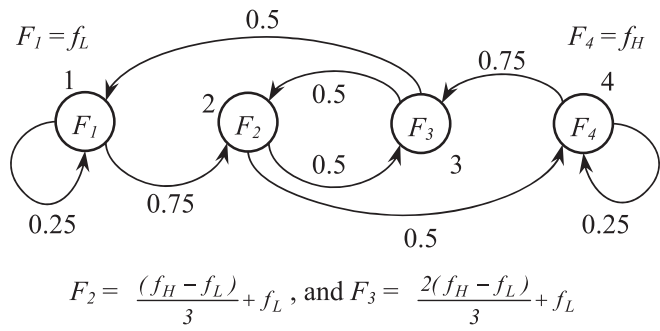

Fig. 8 Switching frequency modulation governed by Markov chain.

the state of a Markov chain [13], [14]. The introduced example in [13] is applied here for comparing the EMI performances. Figure 8 schematically shows a four state Markov chain, corresponding to the following policy. The controller observes the last switching frequency and if it is $F_{1}$, then either of $F_{1}$ with probability 0.25 or $F_{2}$ with probability 0.75 is selected for the next cycle. If it is $F_{2}$, then either of $F_{3}$ or $F_{4}$ is selected for the next cycle with probability 0.5 . If it is $F_{3}$, then either of $F_{2}$ or $F_{1}$ is selected for the next cycle with probability 0.5 . If it is $F_{4}$, then either of $F_{4}$ with probability 0.25 or $F_{3}$ with probability 0.75 is selected for the next cycle.

\subsubsection{Uniformly Distributed Random Modulation}

The switching frequency is changed uniformly from $f_{L}$ up to $f_{H}$ at the range of $f_{c s w} \pm \Delta f$ as shown in Fig. 9 . 


\subsubsection{Normally Distributed Random Modulation}

The switching frequency is changed normally from $f_{L}$ up to

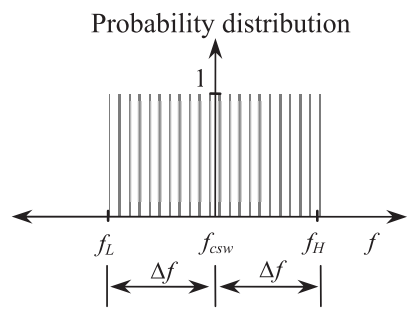

(a)

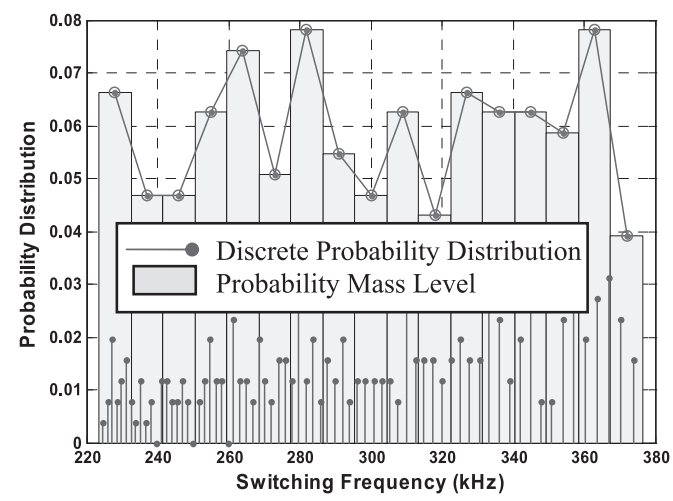

(b)

Fig. 9 Uniformly distributed random modulation: (a) ideal normalized uniformly distributed random sequence and (b) discrete probability distribution and probability mass level for the actual generated sequence.

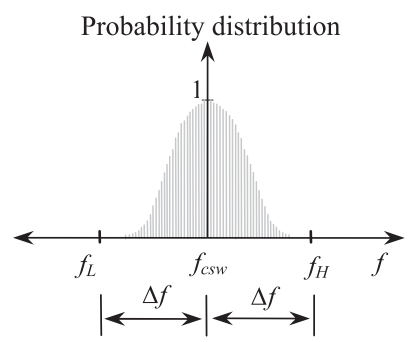

(a)

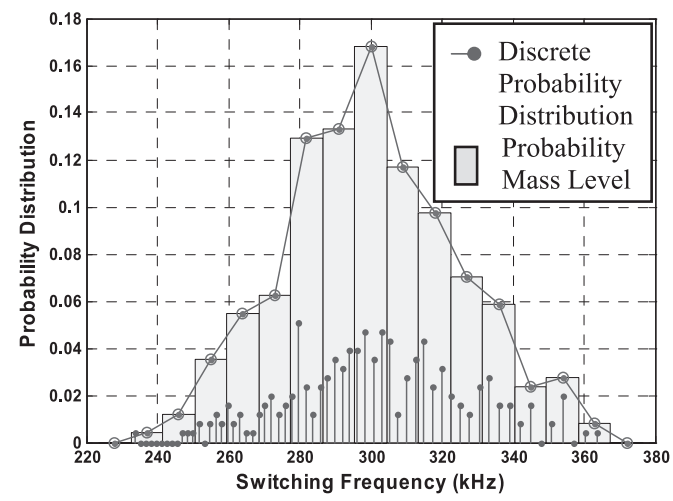

(b)

Fig. 10 Normally distributed random modulation: (a) ideal normalized normally distributed random sequence and (b) discrete probability distribution and probability mass level for the actual generated sequence.
$f_{H}$ at the range of $f_{c s w} \pm \Delta f$ as shown in Fig. 10.

\subsubsection{M-Sequence Based Random Modulation}

In this case, the modulated frequency is shifted between two values $\left(f_{L}\right.$ and $\left.f_{H}\right)$ corresponding to the output signal of a 16-bit M-sequence (' 0 ' and ' 1 ,' respectively) [15].

\subsubsection{Pseudorandom Stream Modulation}

A pseudorandom stream generator has been constructed using several maximum length linear feedback shift registers (m-LFSRs) in parallel. The using of m-LFSRs is due to the fact that the sequence generated by the m-LFSRs has a maximum period [16]. For different m-LFSRs output bits, different initial contents of m-LFSRs (seeds) have been used. The taps are XOR'd sequentially with the output and then fed back into the leftmost bit.

The designed pseudorandom stream generator delivers a 16-bit output as shown in Fig. 11. The component mLFSRs are clocked regularly. Only at the beginning of every switching cycle, the random output bits are converted into an integer number and used in calculating the switching frequency for the started switching cycle.

\section{Experimental Work}

As described in Fig. 1 and Fig. 12, the line impedance stabilization network (LISN) is used to sense the conductednoise that is measured by an EMI receiver [1].

The generated sequences have been designed and implemented inside an FPGA device. A synchronous buck converter topology was selected. The output ripple voltage has been measured and the produced acoustic noise has been

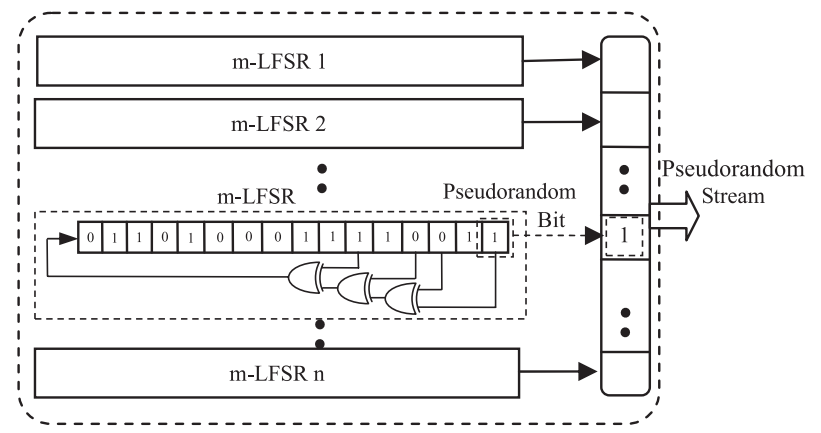

Fig. 11 The proposed pseudorandom stream generator.

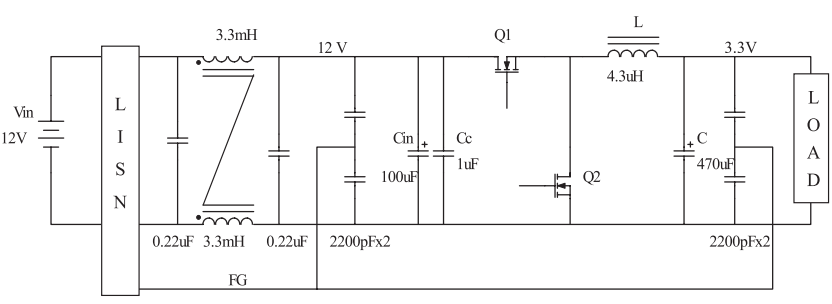

Fig. 12 Experimental converter circuit configuration. 


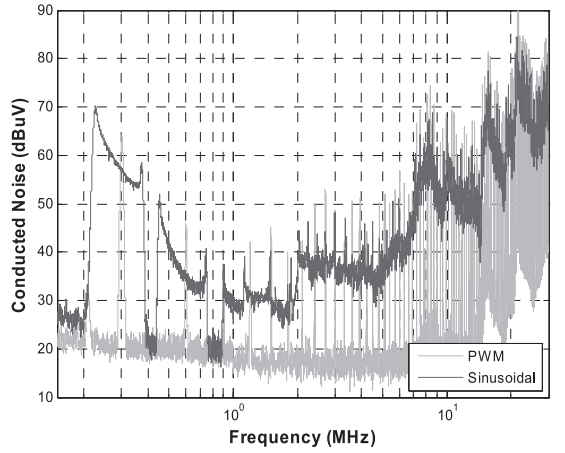

(a)

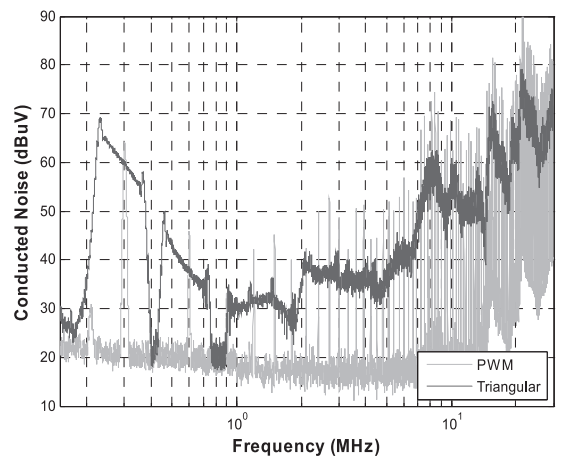

(b)

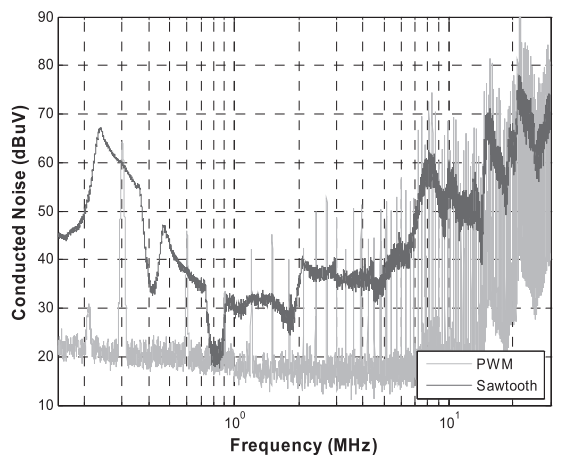

(c)

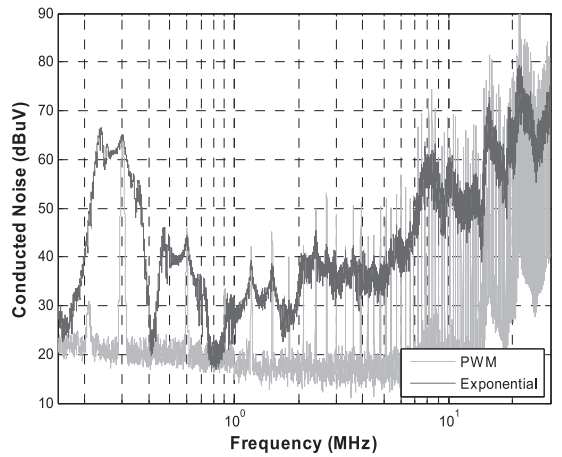

(d)

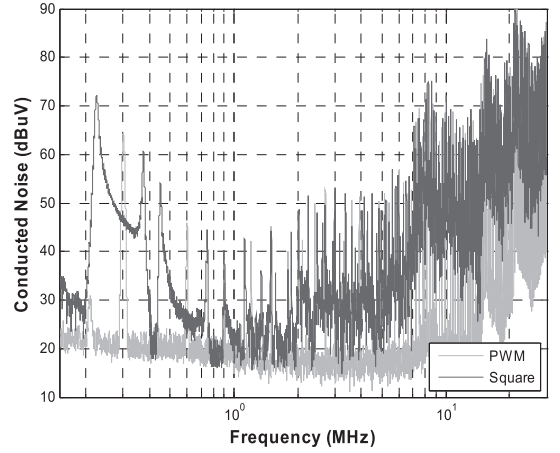

(e)

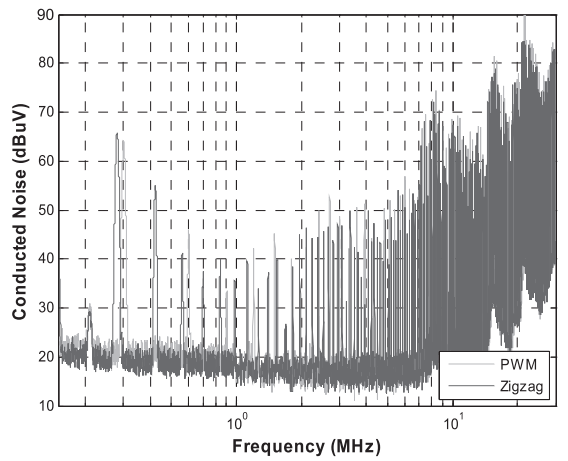

(f)

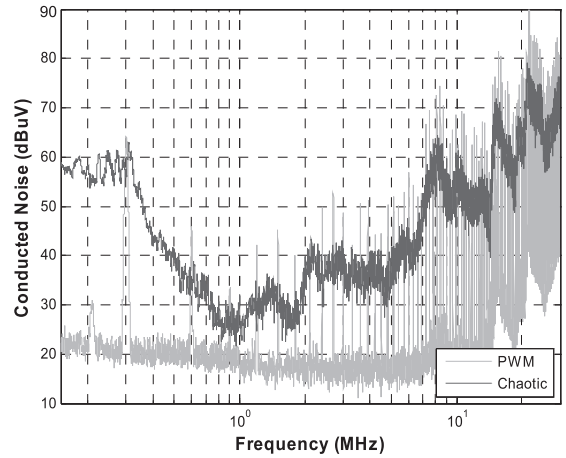

(g)

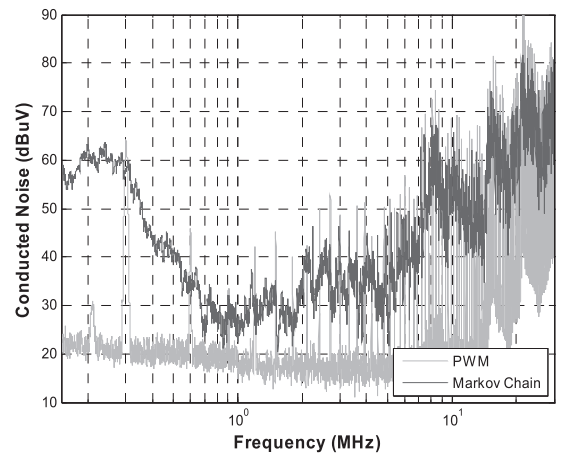

(h)

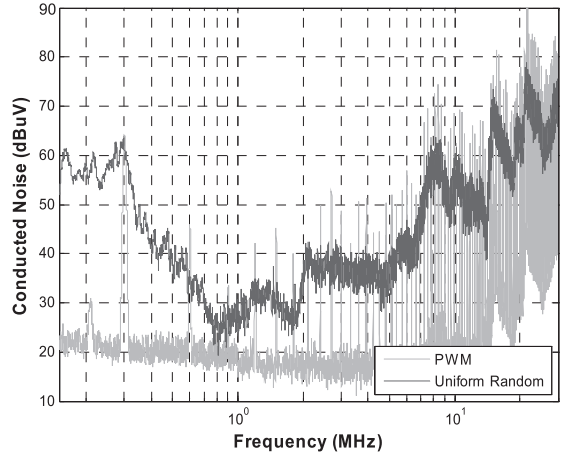

(i)

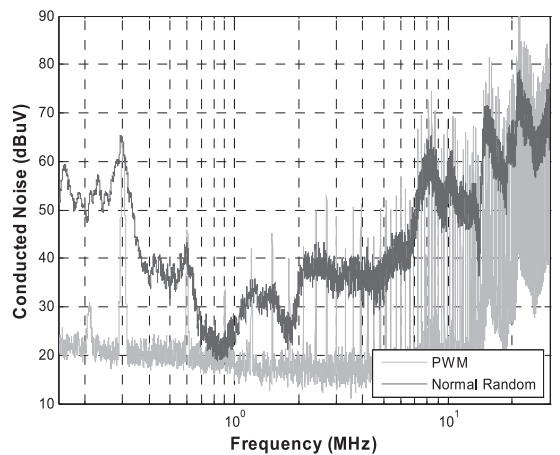

(j)

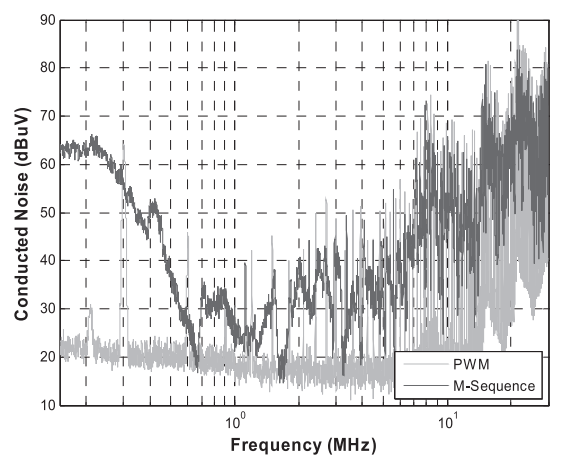

(k)

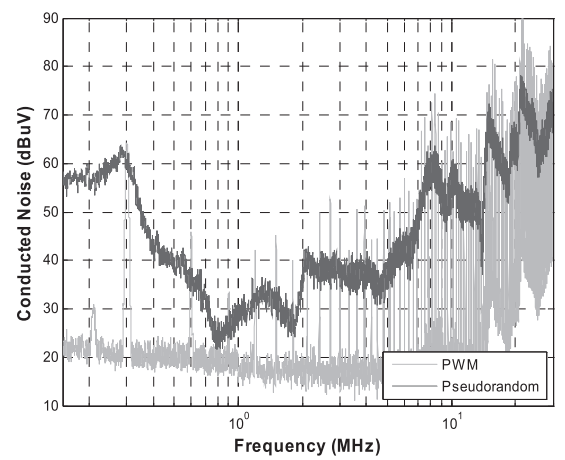

(1)

Fig. 13 Measured conducted-noise frequency spectra using different frequency modulation profiles. 
Table 1 Summary of the experimental results for the frequency modulation regimes with different profiles.

\begin{tabular}{|c|c|c|c|c|c|c|}
\hline \multirow{2}{*}{$\begin{array}{l}\text { Q } \\
\dot{0} \\
\dot{0}\end{array}$} & \multirow{2}{*}{ 赵 } & \multirow{2}{*}{ Frequency modulation profile } & \multicolumn{2}{|c|}{ Noise level reduction, $\mathrm{dB}$} & \multirow{2}{*}{$\begin{array}{l}\text { Percentage increase of ripple voltage } \\
\text { with the reference of that at PWM }\end{array}$} & \multirow{2}{*}{ Acoustic noise } \\
\hline & & & $\mathrm{LF}$ & $\mathrm{HF}$ & & \\
\hline \multirow{6}{*}{ I } & 1 & Sinusoidal modulation & -6.0 & 6.0 & 3.90625 & No \\
\hline & 2 & Triangular modulation & -5.1 & 11.2 & 2.734375 & No \\
\hline & 3 & Sawtooth modulation & -3.0 & 12.5 & 3.125 & Small \\
\hline & 4 & Exponential modulation & -2.2 & 11.0 & 0.78125 & No \\
\hline & 5 & Square modulation & -7.8 & -0.2 & 3.90625 & Large \\
\hline & 6 & Zigzag modulation & -1.7 & 5.8 & 0 & No \\
\hline \multirow{6}{*}{ II } & 7 & Chaotic modulation & 1.2 & 11.3 & 0.78125 & Small \\
\hline & 8 & Markov chain based random modulation & 0.5 & 8.8 & 1.5625 & Large \\
\hline & 9 & Uniformly distributed random modulation & 0.8 & 11.4 & $\mathbf{0}$ & No \\
\hline & 10 & Normally distributed random modulation & -1.2 & 11.4 & -0.390625 & Small \\
\hline & 11 & M-sequence based random modulation & -2.1 & 6.5 & 0.78125 & Large \\
\hline & 12 & Pseudorandom stream modulation & 0.5 & 12.3 & $\mathbf{0}$ & No \\
\hline
\end{tabular}

noticed and recorded. Noise was measured at: $V_{\text {in }}=12 \mathrm{~V}$, $V_{o}=3.3 \mathrm{~V}$, and $I_{o}=5 \mathrm{~A}$ at a resolution band width of the spectrum analyzer $\mathrm{RBW}=9 \mathrm{kHz}$.

\section{Results and Discussion}

The measured conducted-noise frequency spectra are shown in Fig. 13 using different frequency modulation profiles.

Although the probability mass levels for the square and zigzag modulations are similar (see Fig. 5(b) and Fig. 6(b)), the accompanied conducted-noise frequency spectra are different (see Fig. 13(e) and Fig. 13(f)). That is due to their different frequency modulation sequence profiles.

Both of the chaotic and uniformly distributed random modulations have similar frequency modulation sequence profiles and probability mass levels (see Fig. 7 and Fig. 9). That leads to similar accompanied conducted-noise frequency spectra (see Fig. 13(g) and Fig. 13(i)).

The pseudorandom modulation attains a good EMI performance. That is due to its good probability characteristics and endless random sequence (i.e. the used pseudorandom number generator digitally simulates the white noise random number generator).

It is clear that the noise peaks are concentrated mainly in two regions: the first region in the low-frequency (LF) range $(0.15-1 \mathrm{MHz})$ around the frequencies which have higher probability mass levels (for example see Fig. 13(a) and Fig. 2(b)) and the other region in the high-frequency (HF) range (1-30 MHz).

A comparison has been carried out by subtracting the conducted-noise peak of the PWM regime from that of the variable frequency modulation regime in the two regions.
Table 1 summarizes the measured experimental results for the frequency modulation regimes with different profiles.

\section{Conclusions}

The effect of using different frequency modulation regimes on the conducted-noise characteristics of a dc-dc converter has been experimentally investigated. It can be concluded that:

1. The conducted-noise spreading depends on both the frequency modulation sequence profile and the statistical characteristics of the sequence. Thus both factors should be considered when designing a programmed switching controller targeted conducted-noise reduction in dc-dc converters.

2. Due to its poor EMI performance and large acoustic noise, the profiles in group (I) are not a good choice for frequency modulation regimes in EMI-sensitive and audible applications. They do not significantly improve the conducted-noise spectrum in the high-frequency range. Moreover, they increase the conducted-noise peak in the low-frequency range.

3. The chaotic modulation, uniformly distributed random modulation, and pseudorandom modulation regimes attain the best EMI performance. They improve the conducted-noise characteristics, not only in the lowfrequency range, but also in the high-frequency range. In addition they do not deteriorate the converter's normal operation. 


\section{References}

[1] C.R. Paul, Introduction to Electromagnetic Compatibility, 2nd ed., pp.49-90, John Wiley \& Sons, 2006.

[2] F. Lin and D.Y. Chen, "Reduction of power supply EMI emission by switching frequency modulation," IEEE Trans. Power Electron., vol.9, no.1, pp.132-137, Jan. 1994.

[3] T. Tanaka, T. Ninomiya, and K. Harada, "Random-switching control in DC-to-DC converters," Proc. IEEE PESC'89, pp.500-507, 1989.

[4] T. Ninomiya, T. Tanaka, H. Kameda, and K. Harada, "Noise reduction of switching-mode power converters by random-switching control," Proc. IPEC-Tokyo'90, pp.1165-1172, 1990.

[5] T. Tanaka, H. Kameda, and T. Ninomiya, "Noise analysis of DCto-DC converter with random-switching control," Proc. IEEE INTELEC'91, pp.283-290, 1991.

[6] G.M. Dousoky, M. Shoyama, and T. Ninomiya, "A novel implementation of an FPGA-based controller for conducted-noise reduction in randomly switched DC-DC converters,' Proc. IEEE APEC'09, pp.65-69, 2009.

[7] G.M. Dousoky, M. Shoyama, and T. Ninomiya, "FPGA-based design and implementation of spread-spectrum schemes for conducted-noise reduction in DC-DC converters," Proc. IEEE ICIT09, pp.527-532, 2009.

[8] G.M. Dousoky, M. Shoyama, and T. Ninomiya, "Triple-hybrid spread-spectrum technique for conducted-noise reduction in DC-DC converters with an FPGA-based implementation," Proc. EPE 2009, ISBN: 9789075815009, pp.1-8, Sept. 2009.

[9] G.M. Dousoky, M. Shoyama, and T. Ninomiya, "Double-hybrid spread-spectrum technique for conducted-EMI reduction in DC-DC switching regulators with FPGA-based controller," Proc. IEEE INTELEC'09, pp.1-6, Oct. 2009.

[10] J. Balcells, A. Santolaria, A. Orlandi, D. Gonzalez, and J. Gago, "EMI reduction in switched power converters using frequency modulation techniques," IEEE Trans. Electromagn. Compat., vol.47, no.3, pp.569-576, Nov. 2005.

[11] A. Santolaria, J. Balcells, D. Gonzalez, J. Gago, and S.D. Gil, "EMI reduction in switched power converters by means of spread spectrum modulation techniques," IEEE PESC'04, pp.292-296, 2004.

[12] A. Santolaria, SSCG methods of EMI emissions reduction applied to switching power converters, Ph.D. dissertation, Dept. Electon. Eng. Universitat Politcnica de Catalunya, 2004.

[13] A.M. Stankovic, G.C. Verghese, and D.J. Perreault, "Randomized modulation schemes for power converters governed by Markov chains," Proc. 4th IEEE Conference on Control Applications, pp.372-377, Sept. 1995.

[14] A.M. Stankovic, "Random pulse modulation with application to power electronic converters," Ph.D. dissertation, Dept. Elect. Eng. Comput. Sci., M.I.T., Cambridge, 1993.

[15] T. Tanaka, H. Hamasaki, and H. Yoshida, "Random-switching control in DC-to-DC converters: An implementation using Msequence," Proc. IEEE INTELEC'97, pp.431-437, 1997.

[16] A.J. Menezes, P.C. van Oorschot, and S.A. Vanstone, Handbook of Applied Cryptography, pp.191-222, CRC Press, 1996.

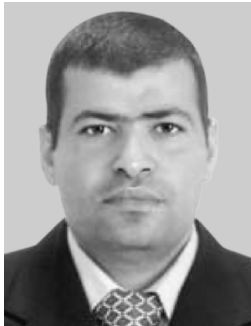

Gamal M. Dousoky was born in Minia Egypt, in 1977. He received his B.Sc. and M.Sc. in Electrical Engineering from Minia University, Minia, Egypt, in 2000 and 2004, respectively. $\mathrm{He}$ is currently working toward his Ph.D. in the Department of Electrical Engineering, Graduate School of Information Science and Electrical Engineering, Kyushu University, Fukuoka, Japan. Since 2000, he has been associated with the Department of Electrical Engineering, Faculty of Engineering at Minia University, first as a Teaching Assistant and, since 2004, as a Lecturer Assistant. His research interests include power electronics, especially renewable energy applications, switching power supplies, EMC/EMI, artificial intelligence, and digital control. Mr. Dousoky is a Student Member of IEEE, IEEE Power Electronics Society (PELS), and IEEE Industrial Electronics Society (IES). He received the 2009 Excellent Student Award from the IEEE Fukuoka Section.

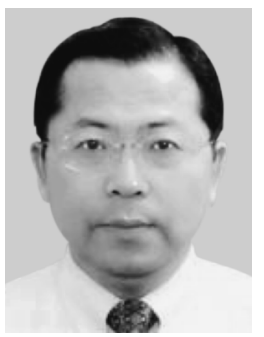

Masahito Shoyama received his B.S. in Electrical Engineering and his Dr.Eng. from Kyushu University, Fukuoka, Japan, in 1981 and 1986, respectively. He joined the Dept. of Electronics, Kyushu University as a Research Associate in 1986. He had been an Associate Professor since 1990, and he has been a Professor since 2010. Since 2009 he has been with the Dept. of Electrical Engineering, Faculty of Information Science and Electrical Engineering, Kyushu University. He has been active in the fields of power electronics, especially in high-frequency switching power supplies, power factor correctors, and electromagnetic compatibility (EMC). Prof. Shoyama is a member of IEEE, IEEJ and SICE.

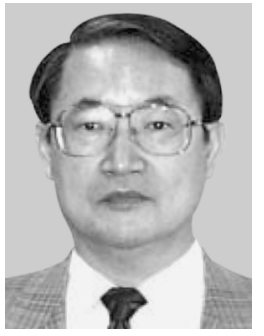

Tamotsu Ninomiya received the B.E., M.E., and Dr.Eng. degrees in electronics from Kyushu University, Fukuoka, Japan, in 1967, 1969, and 1981, respectively. Since 1969 he has been associated with the Department of Electronics, Kyushu University, first as Research Assistant and since 1988 as Professor. Since the reorganization in 1996, he has been a Professor in the Dept. of Electrical and Electronic Systems Engineering of the Graduate School of Information Science and Electrical Engineering. He has been a specialist in the field of power electronics. He served as General Chairman in 1998 PESC, and in January 2001, he was awarded as IEEE Fellow for contributions to the development of high-frequency switching power converters. He also served as Chairman of the Technical Group on Power Engineering in Electronics and Communications of the IEICE, and an Associate Editor of IEICE Transactions on Communications. Currently he is a TDK Endowed Chair Professor at Faculty of Engineering, Nagasaki University. 